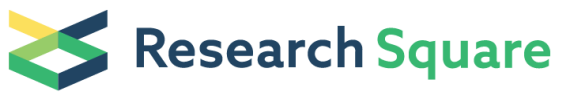 \\ Preprints are preliminary reports that have not undergone peer review. \\ They should not be considered conclusive, used to inform clinical practice, \\ or referenced by the media as validated information.
}

\section{Prevalence and Risk Factors of Metabolic Associated Fatty Liver Disease in China: A Cross-Sectional Study}

\author{
Yuling Chen \\ The First Affiliated Hospital of Chongqing Medical University \\ Hao Li \\ The First Affiliated Hospital of Chongqing Medical University
}

\section{Shu Li}

The First Affiliated Hospital of Chongqing Medical University

Zhou Xu

Affiliated Hospital of North Sichuan Medical College

Shen Tian

The First Affiliated Hospital of Chongqing Medical University

Juan Wu

The First Affiliated Hospital of Chongqing Medical University

Xin-yu Liang

The First Affiliated Hospital of Chongqing Medical University

Xin Li

The First Affiliated Hospital of Chongqing Medical University

Zi-li Liu

The First Affiliated Hospital of Chongqing Medical University

Jun Xiao

The First Affiliated Hospital of Chongqing Medical University

Jia-ying Wei

The First Affiliated Hospital of Chongqing Medical University

Chenyu Ma

The First Affiliated Hospital of Chongqing Medical University

Kai-nan Wu

The First Affiliated Hospital of Chongqing Medical University

Liang Ran

The First Affiliated Hospital of Chongqing Medical University

Lingquan Kong ( $\square$ huihuikp@163.com )

The First Affiliated Hospital of Chongqing Medical University https://orcid.org/0000-0001-5705-9001

Research article

Keywords: Metabolic associated fatty liver disease, Nonalcoholic fatty liver disease, metabolic syndrome, ultrasonography

Posted Date: February 11th, 2021

DOI: https://doi.org/10.21203/rs.3.rs-208721/v1

License: (c) (i) This work is licensed under a Creative Commons Attribution 4.0 International License. Read Full License 


\section{Abstract}

\section{Background}

Metabolic associated fatty liver disease (MAFLD) is a new definition for liver disease associated with known metabolic dysfunction. Based on new diagnostic criteria, we aim to investigate its prevalence and risk factors in Chinese.

\section{Methods}

This cross-sectional study was implemented on 139170 subjects who participated in health examination.

\section{Results}

Among 139170 subjects, prevalence of MAFLD was 26.1\% (males: 35.4\%; females: $14.1 \%$ ), and it was significantly higher before 65 -year-old in males than that in females $(36.2 \%$ vs. $12.2 \%$, $\mathrm{P}<0.001)$, however significantly lower after $65(28.2 \%$ vs. $33.0 \%$, $P<0.001)$. The prevalence in different female menopausal status (premenopause, perimenopause, postmenopause) was $6.1 \%, 16.8 \%$, and 30.2\%, in BMI groups (underweight, normal, overweight and obese), it was $0.1 \%, 4.0 \%, 27.4 \%$ and $59.8 \%$. Proportions of abnormal metabolic features in MAFLD group were significantly higher than those in non-MAFLD group, so as the proportion of elevated alanine aminotransferase (ALT) $(42.5 \%$ vs. $11 \%$, P $<0.001)$. Prevalence of metabolic syndrome (MS), dyslipidemia, and hyperuricemia in MAFLD group (53.2\%, 80.0\%, and 45.0\%) was significantly higher than that in non-MAFLD group (10.1\%, $41.7 \%$, and $16.8 \%$ ). Logistic regression revealed that age, BMI, waist circumference, ALT, triglyceride, fasting glucose, uric acid and platelet count were associated with MAFLD.

\section{Conclusions}

MAFLD is prevalent in China, its prevalence varies among different groups of age, gender, BMI, and female menopausal status. MAFLD was related to metabolic disorders especially obesity. People with MAFLD suffer high prevalence of MS, dyslipidemia, hyperuricemia, and elevated liver enzymes. Metabolic disorders should be paid attention to improve management of MAFLD.

\section{Background}

Metabolic associated fatty liver disease (MAFLD), formerly called nonalcoholic fatty liver disease (NAFLD), is a new definition for liver disease associated with known metabolic dysfunction, which is the most common chronic liver disease around the world. It was reported that NAFLD affected $24 \%$ of the adult population around world, which has posed threat to human health [1]. NAFLD is generally considered to be closely related to obesity and multiple metabolic disorders and it can vary from hepatic steatosis to steatohepatitis, fibrosis or cirrhosis [2], it is regarded as the hepatic manifestation of multisystem metabolic dysfunction [3]. Previously, the diagnosis of NAFLD was an exclusive diagnosis [3], however, since researches has progressed, NAFLD was found to be derived from the potential state of multiple metabolic dysfunction with complex pathophysiological characteristics, and the high prevalence in the general population makes it common to be co-existed with other liver diseases, which indicates that the exclusive criteria can no longer meet current requirement for the diagnosis of the disease. Hence, in recent international expert consensus, "MAFLD" was considered to be a better description of the liver disease associated with known metabolic dysfunction [4], and a set of positive diagnostic criteria were therefore released quickly [5], so that MAFLD can be accurately, comprehensively and easily diagnosed. With the patient population being somewhat different from that of NAFLD, disease characteristics can be better manifested through the patient population diagnosed by new diagnostic criteria.

Therefore, this cross-sectional study aims to investigate the prevalence and risk factors of MAFLD based on new diagnostic criteria, to better elucidate the association between MAFLD and multiple metabolic disorders, so as to provide a more accurate reference for the management and prevention of MAFLD.

\section{Methods}

\section{Study population}

This cross-sectional study used data of a general Chinese population who took part in the health examination at the Quality Control Center of Health Examination in Chongqing (China), which is also known as the Health Management Center of the First Affiliated Hospital of Chongqing Medical University, from January 2015 to September 2018, most of the participants are urban residents. Our study involved 139170 participants which including 78176 males (56.2\%) and 60994 females (43.8\%). All the participants completed comprehensive anthropometric measurements and clinical examination including abdominal ultrasonography, the collection of fasting blood and urine samples. Repeat examinations of the same person were recognized by their unique health examination ID, and only one data set were randomly involved in the study. Exclusion criteria were those with incomplete data; younger than 18 years old; history of malignancy; history of oophorectomy or hysterectomy; history of liver surgery or nephrectomy. The study was approved and supervised by The Ethics Committee of The First Affiliated Hospital of Chongqing Medical University (approval number: 2019-141). 
Blood pressure and anthropometric parameters including height, weight and waist circumference were measured in standardized procedures by trained examiners. Body mass index (BMI) was calculated as follows: BMI $\left(\mathrm{kg} / \mathrm{m}^{2}\right)=$ weight $(\mathrm{kg}) / \mathrm{height}$ squared ( $\left.\mathrm{m}^{2}\right)$. Venous blood samples of all participants were collected after at least 8 hours of fasting and were analyzed by standard procedures in the laboratory of First Affiliated Hospital of Chongqing Medical University, which was certified by the College of American Pathologists (CAP No.: 7215494). Abdominal ultrasound was performed using ultrasound scanners (Aplio500, Toshiba Medical Systems, Japan or HD11XE, Philips Medical Systems, U.S.A). All the abdominal ultrasonography was performed and evaluated by experienced ultrasonographist in the Quality Control Center of Health Examination. Since the diagnosis of MAFLD does not involve the assessment of alcohol consumption and hepatitis virus, here we didn't include the two examinations in our study. Disease histories were checked in the health examination results of each participant. All data were recorded in the electronic medical record system of the Quality Control Center of Health Examination in Chongqing.

\section{Diagnosis of MAFLD}

The diagnosis of MAFLD is based on the ultrasonically diagnosed hepatic steatosis and the presence of one of the following three criteria: overweight or obesity (defined as $\mathrm{BMI} \geq 23 \mathrm{~kg} / \mathrm{m}^{2}$ in Asians), type 2 diabetes mellitus, or metabolic dysregulation. Metabolic dysregulation is defined by the presence of at least two following metabolic risk abnormalities: 1) Waist circumference $\geq 90 / 80 \mathrm{~cm}$ in Asian men and women; 2) Blood pressure $\geq 130 / 85 \mathrm{mmHg}$ or specific drug treatment; 3) Plasma triglycerides $\geq 1.70 \mathrm{mmol} / \mathrm{L}$ or specific drug treatment; 4) Plasma HDL-cholesterol $<1.0$ $\mathrm{mmol} / \mathrm{L}$ for men and $<1.3 \mathrm{mmol} / \mathrm{L}$ for women or specific drug treatment; 5) Prediabetes (i.e., fasting glucose levels 5.6 to $6.9 \mathrm{mmol} / \mathrm{L}$, or 2 -hour postload glucose levels 7.8 to 11.0 mmol or HbA1c $5.7 \%$ to $6.4 \%$; 6) Plasma high-sensitivity C-reactive protein (hs-CRP) level >2 mg/L; 7) Homeostasis model assessment (HOMA)-insulin resistance score $\geq 2.5$ [5]. The diagnosis of hepatic steatosis on ultrasound was based on the wildly used criteria [6].

\section{Definitions}

BMI groups of underweight ( $<18.5 \mathrm{~kg} / \mathrm{m} 2)$, normal $(\geq 18.5 \mathrm{~kg} / \mathrm{m} 2,<23.0 \mathrm{~kg} / \mathrm{m} 2)$, overweight $(\geq 23.0 \mathrm{~kg} / \mathrm{m} 2,<25.0 \mathrm{~kg} / \mathrm{m} 2)$ and obese $(\geq 25.0 \mathrm{~kg} / \mathrm{m} 2)$ were categorized based on the BMI criteria for Asians made by WHO [7]. Metabolic syndrome (MS) was defined in accordance with the criteria by Joint Statement [8], which was based on the presence of at least 3 of the following components: 1) Elevated waist circumference ( $\geq 90 \mathrm{~cm}$ for men and $\geq 80 \mathrm{~cm}$ for women); 2) Elevated triglycerides ( $\geq 1.70 \mathrm{mmol} / \mathrm{L}$ ) or the drug treatment for elevated triglycerides; 3 ) Reduced HDL-C (<1.0 mmol/L for men and $<1.3 \mathrm{mmol} / \mathrm{L}$ for women) or the drug treatment for reduced HDL-C; 4 ) Elevated blood pressure ( $\geq 130 / 85 \mathrm{~mm} \mathrm{Hg})$ or the drug treatment for the hypertension; 5) Elevated fasting glucose ( $\geq 5.6 \mathrm{mmol} / \mathrm{L})$ or the drug treatment for elevated glucose. Dyslipidemia was defined according to the guidelines for the prevention and treatment of dyslipidemia in Chinese adults [9], as follows: the total cholesterol level of $\geq 5.2 \mathrm{mmol} / \mathrm{L}$; the LDL-C level of $\geq 3.4 \mathrm{mmol} / \mathrm{L}$; the HDL-C level of $<1 \mathrm{mmol} / \mathrm{L}$ and the triglyceride level of $\geq 1.7 \mathrm{mmol} / \mathrm{L}$. Hyperuricemia was defined as the uric acid level of $\geq 416 \mu \mathrm{mol} / \mathrm{L}$ for men or $\geq 357 \mu \mathrm{mol} / \mathrm{L}$ for women [10]. Menopausal status was defined as premenopausal period ( $\leq 45 \mathrm{years}$ old), perimenopausal period (45-54 years old) and postmenopausal period ( $\geq 55$ years old) according to the mean menopausal period for Chinese female population [11, 12]. Elevated liver enzymes was defined as ALT> 35IU/L and AST> 40IU/L [13].

\section{Statistical analysis}

All the continuous variables were tested by normality testing and were described by medians (interquartile range) and proportions. Mann-Whitney $U$ test was performed to compare continuous variables due to their non-normal distribution. For categorical variables, the chi-square test was performed to compare between different variables. Specific prevalence of different age, BMI and female menopausal status groups and their $95 \%$ confidence intervals (Cls)were calculated. Binary logistic regression analysis was performed to explore the related risk factors of MAFLD. Odds ratios (ORs) and their $95 \%$ Cls were finally calculated. Binary logistic regression was performed with RStudio version 4.0.1, and other analysis was performed with SPSS 25. A two-tailed $p$-value $<0.05$ was considered statistically significant.

\section{Results}

\section{General data of 139170 participants.}

Of the 139170 Chinese adults enrolled in the study, 78176 subjects (56.2\%) were males and 60994 (43.8\%) were females. Baseline characteristics of study subjects were showed in Table 1. Compared to people without MAFLD, those with MAFLD were older, predominantly male, had higher values of body mass index (BMI), waist circumference, blood pressure, fasting glucose, total cholesterol, triglyceride, low-density lipoprotein cholesterol (LDL-C), albumin, total bilirubin, alanine aminotransferase (ALT), aspartate aminotransferase (AST), blood urea nitrogen (BUN), creatinine, uric acid, white blood cell count (WBC), red blood cell count (RBC), hemoglobin, platelet count, and hematokrit (HCT), and had lower value of high-density lipoprotein cholesterol (HDL-C) $(P<0.05)$. Compared to males with MAFLD, females with MAFLD tended to be older, had higher values of systolic pressure, fasting glucose, total cholesterol, HDL-C LDL-C, and platelet count. 
Baseline characteristics of the study participants

\begin{tabular}{|c|c|c|c|c|c|c|c|c|c|c|}
\hline \multirow[t]{2}{*}{ Characteristics } & \multicolumn{3}{|c|}{ Total $(\mathrm{N}=139170)$} & \multicolumn{3}{|c|}{ Male $(N=78176)$} & \multicolumn{4}{|c|}{ Female $(\mathrm{N}=60994)$} \\
\hline & $\begin{array}{l}\text { MAFLD (N } \\
=36306)\end{array}$ & $\begin{array}{l}\text { Non-MAFLD } \\
(\mathrm{N}= \\
102864)\end{array}$ & $\begin{array}{l}P \text { - } \\
\text { value }\end{array}$ & $\begin{array}{l}\text { MAFLD (N } \\
=27684)\end{array}$ & $\begin{array}{l}\text { Non-MAFLD } \\
(\mathrm{N}=50492)\end{array}$ & $\begin{array}{l}P \text { - } \\
\text { value }\end{array}$ & $\begin{array}{l}\text { MAFLD (N } \\
=8622)\end{array}$ & $\begin{array}{l}\text { Non-MAFLD } \\
(\mathrm{N}=52372)\end{array}$ & $\begin{array}{l}P \text { - } \\
\text { value }\end{array}$ & $\begin{array}{l}P \text { - } \\
\text { value }^{\dagger}\end{array}$ \\
\hline Age (years) & 47(19) & $42(21)$ & $<.001$ & $45(18)$ & $43(22)$ & $\begin{array}{l}< \\
0.001\end{array}$ & $54(19)$ & $41(20)$ & $\begin{array}{l}< \\
0.001\end{array}$ & $<.001$ \\
\hline $\begin{array}{l}\text { Gender, male } \\
\text { (\%) }\end{array}$ & $\begin{array}{l}27684 \\
(76.3)\end{array}$ & $\begin{array}{l}50492 \\
(49.1)\end{array}$ & $<.001$ & - & - & - & - & - & - & - \\
\hline BMI $\left(\mathrm{Kg} / \mathrm{m}^{2}\right)$ & $26.18(3.41)$ & $22.27(3.69)$ & $<.001$ & $26.35(3.24)$ & $23.05(3.43)$ & $\begin{array}{l}< \\
0.001\end{array}$ & $25.59(3.70)$ & 21.48(3.35) & $<.001$ & $\begin{array}{l}< \\
0.001\end{array}$ \\
\hline $\begin{array}{l}\text { Waist } \\
\text { circumference } \\
\text { (cm) }\end{array}$ & $89(9)$ & $78(12)$ & $<.001$ & $91(9)$ & $82(10)$ & $<.001$ & $84(9)$ & $73(9)$ & $\begin{array}{l}< \\
0.001\end{array}$ & $<.001$ \\
\hline $\begin{array}{l}\text { Systolic } \\
\text { pressure } \\
(\mathrm{mmHg})\end{array}$ & $130(24)$ & $117(22)$ & $<.001$ & $130(22)$ & $121(21)$ & $<.001$ & 132(28) & $112(21)$ & $<.001$ & $<.001$ \\
\hline $\begin{array}{l}\text { Diastolic } \\
\text { pressure } \\
(\mathrm{mmHg})\end{array}$ & $81(15)$ & 72(14) & $<.001$ & $82(15)$ & 75(15) & $<0.001$ & $78(16)$ & $69(13)$ & $\begin{array}{l}< \\
0.001\end{array}$ & $<.001$ \\
\hline $\begin{array}{l}\text { Fasting } \\
\text { glucose } \\
\text { (mmol/L) }\end{array}$ & $5.5(1.0)$ & $5.1(0.7)$ & $<.001$ & $5.5(1.0)$ & $5.2(0.6)$ & $<.001$ & $5.6(1.0)$ & $5.1(0.6)$ & $<.001$ & $<.001$ \\
\hline $\begin{array}{l}\text { Total } \\
\text { cholesterol } \\
\text { (mmol/L) }\end{array}$ & $5.02(1.22)$ & $4.64(1.16)$ & $<.001$ & $4.98(1.20)$ & $4.68(1.13)$ & $\hat{0} 0.001$ & $5.13(1.30)$ & $4.61(1.18)$ & $<.001$ & $<.001$ \\
\hline $\begin{array}{l}\text { Triglyceride } \\
\text { (mmol/L) }\end{array}$ & $2.04(1.43)$ & $1.10(0.72)$ & $<.001$ & $2.12(1.50)$ & $1.26(0.83)$ & $<.001$ & $1.79(1.13)$ & $0.97(0.58)$ & $<.001$ & $<.001$ \\
\hline $\begin{array}{l}\mathrm{HDL}-\mathrm{C} \\
\text { (mmol/L) }\end{array}$ & $1.20(0.36)$ & $1.47(0.45)$ & $<.001$ & $1.15(0.32)$ & $1.34(0.39)$ & $<.001$ & $1.35(0.39)$ & $1.60(0.43)$ & $<.001$ & $<.001$ \\
\hline $\begin{array}{l}\text { LDL-C } \\
\text { (mmol/L) }\end{array}$ & $3.30(1.07)$ & $2.83(1.05)$ & $<.001$ & $3.29(1.03)$ & $2.97(1.03)$ & $<.001$ & $3.32(1.14)$ & $2.70(1.04)$ & $<.001$ & 0.001 \\
\hline Albumin $(\mathrm{g} / \mathrm{L})$ & $47(4)$ & $47(4)$ & $<.001$ & $48(4)$ & $47(3)$ & $\begin{array}{l}< \\
0.001\end{array}$ & $46(4)$ & $46(3)$ & $\begin{array}{l}< \\
0.001\end{array}$ & $<.001$ \\
\hline $\begin{array}{l}\text { Total bilirubin } \\
\text { ( } \mu \mathrm{mol} / \mathrm{L})\end{array}$ & $12.3(5.9)$ & $12.2(5.8)$ & 0.002 & $12.8(6.1)$ & 13.3(6.3) & $<.001$ & $10.8(4.7)$ & $11.3(5.0)$ & $<.001$ & $<.001$ \\
\hline ALT (U/L) & $32(24)$ & $18(13)$ & $\dot{0} 001$ & $35(26)$ & $22(15)$ & $<.001$ & $24(16)$ & $15(8)$ & $<.001$ & $<.001$ \\
\hline AST (U/L) & $24(10)$ & $20(7)$ & $<.001$ & $25(10)$ & $22(8)$ & $\begin{array}{l}< \\
0.001\end{array}$ & $22(8)$ & $19(6)$ & $\begin{array}{l}< \\
0.001\end{array}$ & $<.001$ \\
\hline BUN (mmol/L) & $5.1(1.6)$ & $4.9(1.7)$ & $<.001$ & $5.2(1.6)$ & $5.2(1.7)$ & 0.024 & $5.0(1.7)$ & $4.6(1.6)$ & $\begin{array}{l}< \\
0.001\end{array}$ & $<.001$ \\
\hline $\begin{array}{l}\text { Creatinine } \\
\text { ( } \mu \mathrm{mol} / \mathrm{L})\end{array}$ & $73(19)$ & $66(23)$ & $<.001$ & $77(15)$ & $78(15)$ & $<.001$ & $56(12)$ & $56(12)$ & 0.059 & $<.001$ \\
\hline $\begin{array}{l}\text { Uric acid } \\
\text { ( } \mu \mathrm{mol} / \mathrm{L})\end{array}$ & $393(121)$ & $313(115)$ & $<.001$ & $414(110)$ & $368(96)$ & $<.001$ & $323(89)$ & 268(73) & $<.001$ & $<.001$ \\
\hline WBC $\left(\times 10^{9} / \mathrm{L}\right)$ & $6.55(1.97)$ & $5.89(1.85)$ & $\dot{0} 001$ & $6.64(1.96)$ & $6.09(1.88)$ & $\begin{array}{l}< \\
0.001\end{array}$ & $6.29(1.94)$ & $5.71(1.79)$ & $\begin{array}{l}< \\
0.001\end{array}$ & < 0.001 \\
\hline $\operatorname{RBC}\left(\times 10^{12} / \mathrm{L}\right)$ & $5.09(0.62)$ & $4.77(0.70)$ & $\begin{array}{l}< \\
0.001\end{array}$ & $5.20(0.49)$ & $5.12(0.50)$ & $\begin{array}{l}< \\
0.001\end{array}$ & $4.60(0.44)$ & $4.49(0.43)$ & $\begin{array}{l}< \\
0.001\end{array}$ & $\begin{array}{l}<.001 \\
0.00\end{array}$ \\
\hline
\end{tabular}

${ }^{\dagger}$ The $P$-value were calculated from the comparation between the group of Male with MAFLD (27684) and Female with MAFLD (8622).

Data were described by medians (interquartile range) and proportions (\%). P-values were derived from Mann-Whitney $U$ test or chi-square test.

MAFLD, Metabolic dysfunction-associated fatty liver disease; BMI, body mass index; HDL-C, high-density lipoprotein cholesterol; LDL, low-density lipoprotein cholesterol; ALT, alanine aminotransferase; AST, aspartate aminotransferase; BUN, blood urea nitrogen; WBC, white blood cell count; RBC, red blood cell count. HCT, hematokrit; MS, metabolic syndrome. 


\begin{tabular}{|c|c|c|c|c|c|c|c|c|c|c|}
\hline \multirow{2}{*}{$\begin{array}{l}\text { Characteristics } \\
\text { Hemoglobin } \\
(\mathrm{g} / \mathrm{L})\end{array}$} & \multicolumn{3}{|c|}{ Total $(\mathrm{N}=139170)$} & \multicolumn{3}{|c|}{ Male $(N=78176)$} & \multicolumn{4}{|c|}{ Female $(\mathrm{N}=60994)$} \\
\hline & 155(18) & 144(22) & $<.001$ & 159(14) & 157(13) & $<.001$ & 138(11) & 134(12) & $\begin{array}{l}< \\
0.001\end{array}$ & $\stackrel{<}{0.001}$ \\
\hline $\begin{array}{l}\text { Platelet count } \\
\left(\times 10^{9} / \mathrm{L}\right)\end{array}$ & $211(75)$ & $210(73)$ & $\begin{array}{l}< \\
0.001\end{array}$ & 207(72) & 200(69) & $\begin{array}{l}< \\
0.001\end{array}$ & 226(82) & 219(76) & $\begin{array}{l}< \\
0.001\end{array}$ & $<0.001$ \\
\hline НСТ (\%) & $45.8(4.8)$ & $43.1(6.0)$ & $\begin{array}{l}< \\
0.001\end{array}$ & $46.8(3.6)$ & $46.3(3.7)$ & $\begin{array}{l}< \\
0.001\end{array}$ & 41.5(3.3) & $40.6(3.3)$ & $\begin{array}{l}< \\
0.001\end{array}$ & $\begin{array}{l}< \\
0.001\end{array}$ \\
\hline MS (\%) & 19310(53.2) & $10340(10.1)$ & $<.001$ & 14013(50.6) & $5675(11.2)$ & $<.001$ & $5297(61.4)$ & $4665(8.9)$ & $\begin{array}{l}< \\
0.001\end{array}$ & $<$ \\
\hline $\begin{array}{l}\text { Dyslipidemia } \\
\text { (\%) }\end{array}$ & $29027(80.0)$ & $42854(41.7)$ & $<_{0.001}$ & 22600(81.6) & 24797(49.1) & $\begin{array}{l}<.001 \\
0.0\end{array}$ & 6427(74.5) & $18057(34.5)$ & $<0.001$ & $\dot{0}_{0.001}$ \\
\hline $\begin{array}{l}\text { Hyperuricemia } \\
\text { (\%) }\end{array}$ & $16325(45.0)$ & 17314(16.8) & $<.001$ & 13605(49.1) & $13175(26.1)$ & $<.001$ & $2720(31.5)$ & $4139(7.9)$ & $\begin{array}{l}< \\
0.001\end{array}$ & $\begin{array}{l}< \\
0.001\end{array}$ \\
\hline \multicolumn{11}{|c|}{${ }^{\dagger}$ The $P$-value were calculated from the comparation between the group of Male with MAFLD (27684) and Female with MAFLD (8622). } \\
\hline \multicolumn{11}{|c|}{ Data were described by medians (interquartile range) and proportions (\%). P-values were derived from Mann-Whitney U test or chi-square test. } \\
\hline \multicolumn{11}{|c|}{$\begin{array}{l}\text { MAFLD, Metabolic dysfunction-associated fatty liver disease; BMI, body mass index; HDL-C, high-density lipoprotein cholesterol; LDL, low-density } \\
\text { lipoprotein cholesterol; ALT, alanine aminotransferase; AST, aspartate aminotransferase; BUN, blood urea nitrogen; WBC, white blood cell count; } \\
\text { RBC, red blood cell count. HCT, hematokrit; MS, metabolic syndrome. }\end{array}$} \\
\hline
\end{tabular}

\section{Prevalence of MAFLD and the stratification of age, sex, menopausal status, and BMI}

Of the 139170 participants, $36306(26.1 \%)$ were diagnosed with MAFLD and there was significant difference between males and females in the prevalence of MAFLD (35.4\% vs. $14.1 \%, P<0.001)$. After adjusted by age and sex, the overall prevalence of MAFLD was $23.8 \%$ (males: $32.3 \%$ \& females: 13.4\%). Age-specific prevalence of MAFLD was shown in Fig. 1. The prevalence tended to rise with the increase of age and then decrease, with the peak total prevalence being reached at $34.5 \%$ in the age range of 55-59. For females, the prevalence of MAFLD rose slowly between the age range of $18-49$, however it rose steeply after 50 years, which was consistent with the appearance of perimenopausal period, and peaked at $35.2 \%$ in the age range of $65-69$, while for males, the prevalence rose rapidly between the age of $18-39$, then rose slowly after 40 years and it peaked at $42.5 \%$ in the age range of 50-54, revealing that the peak prevalence appeared earlier in males than that in females. The prevalence of MAFLD was significantly higher before the age of 65 in males than that in females $\left(36.2 \%\right.$ vs. $12.2 \%, \chi^{2}$ value $\left.=9378.514, P<0.001\right)$, while after the age of 65 , it was significantly lower than that in females $\left(28.2 \%\right.$ vs. $33.0 \%, \chi^{2}$ value $\left.=35.532, P<0.001\right)$. The prevalence of MAFLD in different groups of menopausal status in females was $6.1 \%$ [Cl 5.9\%-6.4\%] for the premenopausal period, $16.8 \%$ [Cl $16.2 \%-17.4 \%$ ] for the perimenopausal period and $30.2 \%$ [Cl 29.4\%-30.9\%] for the postmenopausal period, respectively, and there was significant difference in the prevalence between different groups $\left(X^{2}\right.$ value $\left.=4764.496, P<0.001\right)$ (Fig. 2). The prevalence of MAFLD in different BMI groups was $0.1 \%$ [Cl $\left.0.1 \%-0.2 \%\right]$ for the underweight group, $4.0 \%$ [Cl 3.8\%-4.1\%] for the normal group, 27.4\% [Cl 26.9\%-27.9\%] for the overweight group and 59.8\% [Cl 59.3\%-60.3\%] for the obese group, respectively, and there was significant difference between different BMI groups $\left(\chi^{2}\right.$ value $\left.=41904.598, P<0.001\right)$ (Fig. 3).

\section{Related risk factors of MAFLD and association between MAFLD and metabolic disorders.}

The proportion of abnormal metabolic features in MAFLD group were all significantly higher than those in non-MAFLD group $(P<0.001$, Table 2$)$. The proportion of elevated liver enzymes especially elevated ALT was also significantly higher in people with MAFLD than that in people without MAFLD (42.5\% vs. $11.1 \%$ for elevated ALT, $\chi^{2}$ value $\left.=16944.455, P<0.001\right)$. For people with MAFLD, they are more likely to have multiple metabolic disorders, and the prevalence of MS, dyslipidemia and hyperuricemia in people with MAFLD were all significantly higher than that in people without MAFLD (Fig. 4) (For MS: 53.2\% [Cl 52.7\%-53.7\%] vs. 10.1\% [CI 9.9\%-10.2\%], $\chi^{2}$ value $=29779.866, P<0.001$. For dyslipidemia: 80.0\% [CI 79.5\%-80.4\%] vs. 41.7\% [Cl 41.4\%-42.0\%], $\chi^{2}$ value $=15754.446, P<0.001$. For hyperuricemia: $45.0 \%$ [Cl 44.5\%-45.5\%] vs. $16.8 \%[C l 16.6 \%-17.1 \%], \chi^{2}$ value $=11587.748$, $P<0.001)$, respectively. Notably, for males with MAFLD, the prevalence of dyslipidemia and hyperuricemia was significantly higher than that in females with MAFLD (For dyslipidemia: 81.6\% [Cl 81.2\%-82.1\%] vs. 74.5\% [Cl 73.6\%-75.5\%], $\chi^{2}$ value $=206.391, P<0.001$. For hyperuricemia: 49.1\% [Cl $48.6 \%-49.7 \%$ ] vs. $31.6 \%$ [Cl 30.6\%-32.5\%], $\chi^{2}$ value $\left.=822.636, P<0.001\right)$, while the prevalence of MS in males with MAFLD was significantly lower than that in females with MAFLD (50.6\% [Cl 50.0\%-51.2\%] vs. 61.4\% [Cl 60.4\%-62.5\%], $\chi^{2}$ value $\left.=309.026, \mathrm{P}<0.001\right)$. Moreover, prevalence of MAFLD increased with the increased number of MS components people have (from 3.3\% for 0 components to $82.5 \%$ for all 5 components) (Table 3 ), and for people with and without MS, there was also significant difference in the prevalence of MAFLD (65.1\% [CI 64.6\%-65.7\%] vs. 15.5\% [CI 15.3\%-15.7\%], $\chi^{2}$ 
value $=29779.866, P<0.001)$. In the binary logistic regression, our results revealed that eight variables are closely related to MAFLD, including age, $\mathrm{BMI}$, waist circumference, ALT, triglyceride, fasting glucose, uric acid and platelet count (Table 4). Among them, triglyceride, BMI and fasting glucose were found to have the most significant association with MAFLD, with the highest OR values of $1.776,1.476$ and 1.403 , respectively.

Table 2

Proportions of abnormal metabolic features and elevated liver enzymes among 139170 participants

\begin{tabular}{|c|c|c|c|c|c|c|c|c|}
\hline \multirow[t]{2}{*}{ Characteristics } & \multicolumn{3}{|c|}{ Total $(\mathrm{N}=139170)$} & \multicolumn{5}{|c|}{ Non-obese subjects $(\mathrm{N}=97079)$} \\
\hline & $\begin{array}{l}\text { With MAFLD } \\
(\mathrm{n}[\%])(\mathrm{N}= \\
36306)\end{array}$ & $\begin{array}{l}\text { Without MAFLD } \\
(\mathrm{n}[\%])(\mathrm{N}= \\
102864)\end{array}$ & $\begin{array}{l}P- \\
\text { value }\end{array}$ & $\begin{array}{l}\text { Non-obese with } \\
\text { MAFLD (n [\%]) (N } \\
=11143)\end{array}$ & $\begin{array}{l}\text { Non-obese without } \\
\text { MAFLD }(\mathrm{n}[\%])(\mathrm{N}= \\
85936)\end{array}$ & $\begin{array}{l}P \text { - } \\
\text { value }\end{array}$ & $\begin{array}{l}\text { Obese with } \\
\text { MAFLD (n [\%]) } \\
(\mathrm{N}=25163)\end{array}$ & $\begin{array}{l}P \text { - } \\
\text { value }^{\dagger}\end{array}$ \\
\hline $\begin{array}{l}\text { Elevated waist } \\
\text { circumference }\end{array}$ & $23038(63.5)$ & $18840(18.3)$ & $<001$ & 3337(29.9) & $8238(9.6)$ & $\begin{array}{l}< \\
0.001\end{array}$ & 19701(78.3) & $\begin{array}{l}< \\
0.001\end{array}$ \\
\hline $\begin{array}{l}\text { Elevated } \\
\text { systolic } \\
\text { pressure }\end{array}$ & 18610(51.3) & 25477(24.8) & $<.001$ & 4999(44.9) & 18163(21.1) & $<.001$ & $13611(54.1)$ & $<.001$ \\
\hline $\begin{array}{l}\text { Elevated } \\
\text { diastolic } \\
\text { pressure }\end{array}$ & 13487(37.1) & $14824(14.4)$ & $<001$ & $3334(29.9)$ & 10158(11.8) & $\begin{array}{l}<.001 \\
0.00\end{array}$ & 10153(40.3) & $\begin{array}{l}<.001 \\
0.01\end{array}$ \\
\hline $\begin{array}{l}\text { Elevated } \\
\text { triglyceride }\end{array}$ & 23574(64.9) & 20044(19.5) & $\begin{array}{l}< \\
0.001\end{array}$ & $7182(64.5)$ & $14143(16.5)$ & $\begin{array}{l}< \\
0.001\end{array}$ & $16392(65.1)$ & 0.204 \\
\hline $\begin{array}{l}\text { Reduced HDL- } \\
\text { C }\end{array}$ & $10042(27.7)$ & $12336(12.0)$ & $\begin{array}{l}< \\
0.001\end{array}$ & $3046(27.3)$ & 9301(10.8) & $\begin{array}{l}< \\
0.001\end{array}$ & 6996(27.8) & 0.359 \\
\hline $\begin{array}{l}\text { Elevated } \\
\text { fasting } \\
\text { glucose }\end{array}$ & $17165(47.3)$ & 21259(20.7) & $\begin{array}{l}< \\
0.001\end{array}$ & $5347(48.0)$ & $15835(18.4)$ & $\begin{array}{l}< \\
0.001\end{array}$ & 11818(47.0) & 0.073 \\
\hline $\begin{array}{l}\text { Elevated total } \\
\text { cholesterol }\end{array}$ & $15287(42.1)$ & $28121(27.3)$ & $\begin{array}{l}< \\
0.001\end{array}$ & 4885(43.8) & $22537(26.2)$ & $\begin{array}{l}< \\
0.001\end{array}$ & $10402(41.3)$ & $\begin{array}{l}< \\
0.001\end{array}$ \\
\hline $\begin{array}{l}\text { Elevated LDL- } \\
\text { C (mmol/L) }\end{array}$ & $16386(45.1)$ & $25543(24.8)$ & $\begin{array}{l}< \\
0.001\end{array}$ & $5114(45.9)$ & 19641(22.9) & $\begin{array}{l}< \\
0.001\end{array}$ & $11272(44.8)$ & 0.052 \\
\hline Elevated ALT & $15426(42.5)$ & $11443(11.1)$ & $\begin{array}{l}< \\
0.001\end{array}$ & $3816(34.2)$ & $8032(9.3)$ & $\begin{array}{l}< \\
0.001\end{array}$ & $11610(46.1)$ & $\begin{array}{l}< \\
0.001\end{array}$ \\
\hline Elevated AST & $3443(9.5)$ & $2593(2.5)$ & $<.001$ & $774(6.9)$ & $1963(2.3)$ & $\begin{array}{l}< \\
0.001\end{array}$ & 2669(10.6) & $\begin{array}{l}< \\
0.001\end{array}$ \\
\hline \multicolumn{9}{|c|}{${ }^{\dagger}$ The $P$-value were calculated from the comparation between the group of Obese with MAFLD (25163) and Non-obese with MAFLD (11143). } \\
\hline \multicolumn{9}{|c|}{ Data were described by proportions (\%). $P$-values were derived from chi-square test. } \\
\hline \multicolumn{9}{|c|}{$\begin{array}{l}\text { Elevated waist circumference: } \geq 90 \mathrm{~cm} \text { for men and } \geq 80 \mathrm{~cm} \text { for women. Elevated systolic pressure: } \geq 130 \mathrm{~mm} \text { Hg. Elevated diastolic pressure: } \\
\geq 85 \mathrm{~mm} \text { Hg. Elevated triglyceride: } \geq 1.70 \mathrm{mmol} / \mathrm{L} \text {. Reduced HDL-C: }<1.0 \mathrm{mmol} / \mathrm{L} \text {. Elevated fasting glucose: } \geq 5.6 \mathrm{mmol} / \mathrm{L} \text {. Elevated total } \\
\text { cholesterol: } \geq 5.2 \mathrm{mmol} / \mathrm{L} \text {. Elevated LDL-C: } \geq 3.4 \mathrm{mmol} / \mathrm{L} \text {. Elevated ALT: }>35 \mathrm{IU} / \mathrm{L} \text {. Elevated AST: }>40 \mathrm{IU} / \mathrm{L} \text {. }\end{array}$} \\
\hline \multicolumn{9}{|c|}{$\begin{array}{l}\text { MAFLD, Metabolic dysfunction-associated fatty liver disease; HDL-C, high-density lipoprotein cholesterol; LDL, low-density lipoprotein cholesterol; } \\
\text { ALT, alanine aminotransferase; AST, aspartate aminotransferase. }\end{array}$} \\
\hline
\end{tabular}

Table 3

The prevalence of MAFLD in people with different number of MS components

\begin{tabular}{|llll|}
\hline Number of MS risk components & Total population & People with MAFLD & Prevalence (\%) \\
\hline 0 & 45255 & 1471 & 3.3 \\
\hline 1 & 36339 & 5218 & 14.4 \\
\hline 2 & 27926 & 10307 & 36.9 \\
\hline 4 & 18353 & 10758 & 58.6 \\
\hline 5 & 9033 & 6685 & 74.0 \\
\hline MAFLD, Metabolic associated fatty liver disease; MS, metabolic syndrome. & \\
\hline
\end{tabular}


Table 4

Results of binary logistic regression of MAFLD and the tested variables

\begin{tabular}{|c|c|c|c|}
\hline Variable & $P$-value & OR & $95 \% \mathrm{Cl}$ of OR \\
\hline Age (years) & 0.015 & 1.018 & $1.001-1.033$ \\
\hline BMI $\left(\mathrm{Kg} / \mathrm{m}^{2}\right)$ & 0.000 & 1.476 & $1.320-1.660$ \\
\hline Waist circumference (cm) & 0.006 & 1.057 & $1.017-1.102$ \\
\hline ALT (U/L) & 0.009 & 1.023 & $1.002-1.043$ \\
\hline Triglyceride (mmol/L) & 0.000 & 1.776 & $1.238-2.257$ \\
\hline Fasting glucose (mmol/L) & 0.000 & 1.403 & $1.116-1.839$ \\
\hline Uric acid $(\mu \mathrm{mol} / \mathrm{L})$ & 0.001 & 1.003 & $1.001-1.006$ \\
\hline Platelet count $\left(\times 10^{9} / \mathrm{L}\right)$ & 0.014 & 1.004 & $1.000-1.007$ \\
\hline
\end{tabular}

\section{Association Between Non-obese People And Mafld}

Among the non-obese population, the prevalence of MAFLD was 11.5\% (males:16.4\% \& females: 6.9\%). The proportions of abnormal metabolic features and elevated liver enzymes in non-obese people with MAFLD were all significantly higher than those in non-obese people without MAFLD ( $P$ $<0.001$ ) (Table 2). Compared with obese people with MAFLD, the proportions of elevated waist circumference, elevated systolic and diastolic pressure and elevated liver enzymes were significantly lower in non-obese people with MAFLD, while the proportion of elevated total cholesterol in non-obese people was significantly higher.

\section{Discussion}

In the present study, the prevalence and risk factors of MAFLD were explored, and the significant difference in the prevalence of MAFLD among different groups of gender, age, BMI and female menopausal status were revealed. To our knowledge, this is the first study focusing on the prevalence and associated metabolic characteristics of MAFLD since the new definition of MAFLD was set up $[4,5]$.

The prevalence of MAFLD was $26.1 \%$ (23.8\% after age and sex adjustment), and it was significantly higher in males than in females (35.4\% vs. $14.1 \%$, and $32.3 \%$ vs. $13.4 \%$ after age and sex adjustment), which was consistent with previous studies of NAFLD in Asian populations [14]. revealing that male gender is still predominate in MAFLD. The total prevalence reached peak at the age of 55-59 (Fig. 1). In males, the prevalence rises steeply between the age of 18-39, then rose slowly after 40 years and reached peak at the age of 50-54, revealing that MAFLD is more prevalent in men in their middle ages and they were at an increasing risk of having MALFD while in a younger age which need to be paid special attention. We observed that older men had a lower prevalence of MAFLD than middle-aged men, the possible reasons might be as follows: Some people died of other disease in their older age as fatty liver can significantly increase overall mortality [15], hence these people cannot be counted in; Compared with old men who usually retire, middle-aged men who are at peak of their careers have much more social engagement like dinner and party to take part in, which might increase their risk of having metabolic disorders. Whereas in females, the prevalence of MAFLD is relatively low before the age of 50 , however, it rose sharply between the age of 50-69 and reached peak at the age of 65-69. Notably, the sharply increasing trend in females was consistent with the presence of perimenopausal period, indicating that change of estrogen levels was an important factor of the rapid increase in the prevalence of MAFLD. Besides, after stratification by menopausal status, the prevalence of MAFLD in females rose steady from premenopausal period (6.1\%) to perimenopausal period (16.8\%), then to postmenopausal period (30.2\%) (Fig. 2). This phenomenon consists with former studies [16], indicating that estrogen might have protective effects against MAFLD in females [17]. Conversely, the decrease of estrogen in perimenopausal and postmenopausal women can lead to fat redistribution and thus causing metabolic disorders including dyslipidemia and glucose intolerance [18], which can also be demonstrated in our study that the prevalence of dyslipidemia (22.9\% for premenopausal period, $51.6 \%$ for perimenopausal period and $68.8 \%$ for postmenopausal period) was parallel with the trend of the MAFLD prevalence stratified by menopausal status. This finding indicated that the perimenopausal and postmenopausal status might be substantial risk factors for MAFLD in women, suggesting estrogen deficiency in perimenopausal and postmenopausal women may also play an important role in the development of MAFLD.

Previous studies have found that presence of NAFLD is closely related to MS components such as obesity, insulin resistance, hypertension and dyslipidemia, and is considered to be the liver manifestation of MS [19]. Our study has also found that after stratified by BMI, the prevalence of MAFLD increased sharply with the increase of BMI, reaching 59.8\% in obese people (Fig. 3). In binary logistic regression (Table 4), BMI and waist circumference were also significantly associated with MAFLD, indicating obesity is closely associated with MAFLD and obesity management should be emphasized as weight loss was proved to reduce steatosis [20]. 
In people with MAFLD, the proportions of the abnormal metabolic features were all significantly higher than those in people without MAFLD (Table 2), confirming that MAFLD is closely associated with MS components including abdominal obesity, hypertension, dyslipidemia, and dysglycemia. Among them, in addition to waist circumference, the most significant difference was found in elevated triglyceride, and triglyceride were also shown to be significantly associated with MAFLD in logistic regression (Table 4), with the highest OR value of 1.776, suggesting that elevated triglyceride may be an important risk factor of MAFLD. Moreover, the difference in the proportion of elevated fasting glucose was also very significant, and fasting glucose was also significantly associated with MAFLD in logistic regression with the OR value of 1.403 , consisting with the previous study that there's correlation between fatty liver and dyslipidemia and dysglycemia [21]. Studies have also shown that NAFLD is not only closely related to the cardiovascular and renal diseases associated with MS, but also precedes the presentation of metabolic derangements [22], considering the high proportions of abnormal metabolic features (Table 2) and the significant high prevalence of MS (53.2\% vs. 10.1\%) in people with MAFLD (Fig. 4) in our study, MAFLD may be a significant risk factor of MS. Notably, we noticed that the prevalence of MS in females with MAFLD was significantly higher than that in males with MAFLD (61.4\% vs. 50.6\%, $P<0.001)$ (Fig. 4), indicating that in patients with MAFLD, females may be more susceptible to MS than males. Furthermore, we also found that the prevalence of MAFLD in people without any MS risk factors was $3.3 \%$, while those met all 5 MS criteria had a prevalence of $82.5 \%$ (Table 3), and the prevalence of MAFLD in people with MS was significantly higher than those without MS ( $65.1 \%$ vs. $15.5 \%, P<0.001)$, which also demonstrated that MS and its components are important risk factors of MAFLD.

It was also shown in our study that the prevalence of dyslipidemia and hyperuricemia was significantly higher in people with MAFLD than those without MAFLD ( $80.0 \%$ vs. $41.7 \%$ for dyslipidemia and $45.0 \%$ vs. $16.8 \%$ for hyperuricemia) (Fig. 4 ). Dyslipidemia is a well-known risk factor for NAFLD [3], and this can also be reflected in the sharp rise of the prevalence of MAFLD in perimenopausal and postmenopausal women in our study, which might be related to the dyslipidemia due to estrogen deficiency (Fig. 2). In binary logistic regression, uric acid was showed to be significantly associated with MAFLD. Previous cross-sectional and prospective studies have found that elevated serum uric acid could independently predict the increased risk of NAFLD, even serum uric acid levels within normal range were closely related to the presence of NAFLD independently [23-25]. Hence, combining the findings in our study with previous studies, serum uric acid might be considered as an independent risk factor of MAFLD.

The present study revealed that people with MAFLD are more likely to have elevated liver enzymes especially elevated ALT compared with those without MAFLD (42.5\% vs. $11.1 \%$ for elevated ALT, $P<0.001$ ) (Table 2), which stands for the higher proportion of abnormal liver function in people with MAFLD, consisting with the previous study that the most common cause of unexplained elevated ALT is NAFLD [26]. Moreover, Table 4 showed that ALT was significantly associated with MAFLD and studies have showed that elevated ALT is associated with the development of NAFLD into steatohepatitis and even liver fibrosis [27], indicating that elevated ALT has an important clinical significance for MAFLD. Platelets are considered to be elevated during inflammation, and studies have found a linear correlation between platelet count and the severity of liver fibrosis in people with NAFLD [28], in our study, we also found that platelet count was significantly associated with MAFLD (Table 4), indicating that platelet count and ALT levels may be used as a reference indicator of MAFLD development and the resulting liver fibrosis.

Although the occurrence of NAFLD is closely related to obesity, non-obese people may also suffer from NAFLD, especially in the Asia-Pacific region [29]. In our study, the proportions of abnormal metabolic features in non-obese people with MAFLD were all significantly higher than those in nonobese people without MAFLD (Table 2), suggesting that metabolic disorders also play an important role in the occurrence of MAFLD in non-obese people. Notably, the proportion of elevated total cholesterol was significantly higher in non-obese people with MAFLD than that in obese people with MAFLD, suggesting total cholesterol may play an important role in the presence of non-obese MAFLD which need to further exploration.

Our study also has certain limitations. First, this is a cross-sectional study, therefore, the natural course of MAFLD and causal relationships can't be determined. There was a large sample size with a wide range of clinical data included in our study, making it possible to adjust for underlying confounding factors. Second, the diagnosis of MAFLD was based on ultrasonography, which might be partially insensitive to mild hepatic steatosis. However, ultrasonography has been widely used in the epidemiological investigations of fatty liver since it is safe, non-invasive, widely available, and has an acceptable sensitivity and specificity in the detection of hepatic steatosis [30], and it was also recommended as the first-line imaging method by the Association for the Study of the Liver (APASL) in the clinical guideline for MAFLD [31]. Third, there might be certain selection bias because the population who participated in health examination included in our study tend to be more concerned about their health. Furthermore, some information was not available from the current data of health examinations, like medication history of participants.

\section{Conclusions}

In conclusion, our study revealed a high prevalence of MAFLD among a general Chinese population, and it varies greatly between different groups of gender, age, BMI and female menopausal status. An increasing prevalence was found to be associated with obesity and multiple metabolic disorders and people with MAFLD suffer a high prevalence of MS, dyslipidemia, and hyperuricemia. The presence of MAFLD and MS interact with each other and they may be the risk factor of each other. Moreover, non-obese people also suffer from MAFLD, and it's also closely related to metabolic disorders. We also confirmed the high proportion of elevated ALT in people with MAFLD. Multiple metabolic disorders especially obesity should be paid great attention to improve the prevention and management of MAFLD. More researches are still needed to determine the potential mechanisms of the occurrence of MAFLD, as well as further relationship and causality between MAFLD and multiple metabolic disorders, which would provide crucial implications for the prevention and treatment of MAFLD. 


\section{Abbreviations}

MAFLD; metabolic-dysfunction-associated fatty liver disease; NAFLD:nonalcoholic fatty liver disease; MS:metabolic syndrome; BMI:body mass index; HDL-C:high-density lipoprotein cholesterol; LDL:low-density lipoprotein cholesterol; ALT:alanine aminotransferase; AST:aspartate aminotransferase; BUN:blood urea nitrogen; WBC:white blood cell count; RBC:red blood cell count. HCT:hematokrit; OR:odds ratio; Cl:confidence interval.

\section{Declarations}

Ethics approval and consent to participate: All procedures in studies involving human participants were performed in accordance with the ethical standards of the institutional research committee (The Ethics Committee of the First Affiliated Hospital of Chongqing Medical University, approval number: 2019-141) and with the 1964 Helsinki Declaration and its later amendments or comparable ethical standards. The informed consent was waived because the information of all the participants was anonymous.

Consent for publication: Not applicable.

Availability of data and materials: The datasets analysed during the current study are not publicly available because all data were recorded in the electronic medical record system of the Quality Control Center of Health Examination in Chongqing. However, they are available from the corresponding author on reasonable request.

Competing interests: The authors declare that they have no competing interests.

Funding: There is no funding for this research.

Authors contributions: Study concept and design, LQK and YLC; Methodology, HL, XYL and XL; Investigation, SL and ZX; data acquisition, ST and JW; data analysis, ZLL, JX and JYW; Visualization, CYM; drafting of the manuscript, YLC; Review and Editing, LR, LQK and KNW. All authors read and approved the final manuscript.

Acknowledgments: This study was supported through provision of data by The Quality Control Center of Health Examination in Chongqing, Chongqing Medical University, China. We thank Dr. Li-ping Liu and Dr. Bo Tu for their help in data recognition and classification regarding abdominal ultrasound reports. There is no funding for this research.

\section{References}

1. Younossi Z, Anstee QM, Marietti M, Hardy T, Henry L, Eslam M, George J, Bugianesi E. Global burden of NAFLD and NASH: trends, predictions, risk factors and prevention. Nat Rev Gastroenterol Hepatol. 2018;15(1):11-20.

2. Matteoni CA, Younossi ZM, Gramlich T, Boparai N, Liu YC, McCullough AJ. Nonalcoholic fatty liver disease: a spectrum of clinical and pathological severity. Gastroenterology. 1999;116(6):1413-9.

3. Chalasani N, Younossi Z, Lavine JE, Charlton M, Cusi K, Rinella M, Harrison SA, Brunt EM, Sanyal AJ. The diagnosis and management of nonalcoholic fatty liver disease: Practice guidance from the American Association for the Study of Liver Diseases. Hepatology. 2018;67(1):32857.

4. Eslam M, Sanyal AJ, George J, International Consensus P. MAFLD: A Consensus-Driven Proposed Nomenclature for Metabolic Associated Fatty Liver Disease. Gastroenterology. 2020;158(7):1999-2014. e1991.

5. Eslam M, Newsome PN, Sarin SK, Anstee QM, Targher G, Romero-Gomez M, Zelber-Sagi S, Wai-Sun Wong V, Dufour JF, Schattenberg JM, et al. A new definition for metabolic dysfunction-associated fatty liver disease: An international expert consensus statement. J Hepatol. 2020;73(1):2029 .

6. Needleman L, Kurtz AB, Rifkin MD, Cooper HS, Pasto ME, Goldberg BB. Sonography of diffuse benign liver disease: accuracy of pattern recognition and grading. AJR Am J Roentgenol. 1986;146(5):1011-5.

7. World Health Organization. Regional Office for the Western P: The Asia-Pacific perspective: redefining obesity and its treatment: Sydney: Health Communications Australia; 2000.

8. Alberti KG, Eckel RH, Grundy SM, Zimmet PZ, Cleeman JI, Donato KA, Fruchart JC, James WP, Loria CM, Smith SC Jr, et al. Harmonizing the metabolic syndrome: a joint interim statement of the International Diabetes Federation Task Force on Epidemiology and Prevention; National Heart, Lung, and Blood Institute; American Heart Association; World Heart Federation; International Atherosclerosis Society; and International Association for the Study of Obesity. Circulation. 2009;120(16):1640-5.

9. The Joint Committee. on The Revision of Guidelines for the Prevention and Treatment of Dyslipidemia in Chines Adults. The Guidelines for the Prevention and Treatment of Dyslipidemia in Chinese Adults (2016 Revision). Chinese Circulation Journal. 2016;31(10):937-50.

10. Fang J, Alderman MH. Serum uric acid and cardiovascular mortality the NHANES I epidemiologic follow-up study, 1971-1992. National Health and Nutrition Examination Survey. JAMA. 2000;283(18):2404-10.

11. Collaborative Group on Hormonal Factors in Breast C. Menarche, menopause, and breast cancer risk: individual participant meta-analysis, including 118964 women with breast cancer from 117 epidemiological studies. Lancet Oncol. 2012;13(11):1141-51. 
12. Yang L, Li L, Millwood IY, Lewington S, Guo Y, Sherliker P, Peters SA, Bian Z, Wu X, Yu M, et al. Adiposity in relation to age at menarche and other reproductive factors among 300000 Chinese women: findings from China Kadoorie Biobank study. Int J Epidemiol. 2017;46(2):502-12.

13. Chalasani N, Aljadhey H, Kesterson J, Murray MD, Hall SD. Patients with elevated liver enzymes are not at higher risk for statin hepatotoxicity. Gastroenterology. 2004;126(5):1287-92.

14. Fan JG. Epidemiology of alcoholic and nonalcoholic fatty liver disease in China. J Gastroenterol Hepatol. 2013;28(Suppl 1):11-7.

15. Simon TG, Roelstraete B, Khalili H, Hagstrom H, Ludvigsson JF. Mortality in biopsy-confirmed nonalcoholic fatty liver disease: results from a nationwide cohort. Gut 2020.

16. Shen L, Fan JG, Shao Y, Zeng MD, Wang JR, Luo GH, Li JQ, Chen SY. Prevalence of nonalcoholic fatty liver among administrative officers in Shanghai: an epidemiological survey. World J Gastroenterol. 2003;9(5):1106-10.

17. Gutierrez-Grobe Y, Ponciano-Rodriguez G, Ramos MH, Uribe M, Mendez-Sanchez N. Prevalence of non alcoholic fatty liver disease in premenopausal, posmenopausal and polycystic ovary syndrome women. The role of estrogens. Ann Hepatol. 2010;9(4):402-9.

18. Suzuki A, Abdelmalek MF. Nonalcoholic fatty liver disease in women. Womens Health (Lond). 2009;5(2):191-203.

19. Grundy SM, Cleeman JI, Daniels SR, Donato KA, Eckel RH, Franklin BA, Gordon DJ, Krauss RM, Savage PJ, Smith SC, Jr. et al: Diagnosis and management of the metabolic syndrome: an American Heart Association/National Heart, Lung, and Blood Institute scientific statement: Executive Summary. Crit Pathw Cardiol 2005, 4(4):198-203.

20. Ueno T, Sugawara H, Sujaku K, Hashimoto O, Tsuji R, Tamaki S, Torimura T, Inuzuka S, Sata M, Tanikawa K. Therapeutic effects of restricted diet and exercise in obese patients with fatty liver. J Hepatol. 1997;27(1):103-7.

21. Speliotes EK, Massaro JM, Hoffmann U, Vasan RS, Meigs JB, Sahani DV, Hirschhorn JN, O'Donnell CJ, Fox CS. Fatty liver is associated with dyslipidemia and dysglycemia independent of visceral fat: the Framingham Heart Study. Hepatology. 2010;51(6):1979-87.

22. Ghouri N, Preiss D, Sattar N. Liver enzymes, nonalcoholic fatty liver disease, and incident cardiovascular disease: a narrative review and clinical perspective of prospective data. Hepatology. 2010;52(3):1156-61.

23. Lee JW, Cho YK, Ryan M, Kim H, Lee SW, Chang E, Joo KJ, Kim JT, Kim BS, Sung KC. Serum uric Acid as a predictor for the development of nonalcoholic Fatty liver disease in apparently healthy subjects: a 5-year retrospective cohort study. Gut Liver. 2010;4(3):378-83.

24. Xu C, Yu C, Xu L, Miao M, Li Y. High serum uric acid increases the risk for nonalcoholic Fatty liver disease: a prospective observational study. PLoS One. 2010;5(7):e11578.

25. Hwang IC, Suh SY, Suh AR, Ahn HY. The relationship between normal serum uric acid and nonalcoholic fatty liver disease. J Korean Med Sci. 2011;26(3):386-91.

26. Clark JM, Brancati FL, Diehl AM. The prevalence and etiology of elevated aminotransferase levels in the United States. Am J Gastroenterol. 2003;98(5):960-7.

27. Ratziu V, Giral P, Charlotte F, Bruckert E, Thibault V, Theodorou I, Khalil L, Turpin G, Opolon P, Poynard T. Liver fibrosis in overweight patients. Gastroenterology. 2000;118(6):1117-23.

28. Yoneda M, Fujii H, Sumida Y, Hyogo H, Itoh Y, Ono M, Eguchi Y, Suzuki Y, Aoki N, Kanemasa K, et al. Platelet count for predicting fibrosis in nonalcoholic fatty liver disease. J Gastroenterol. 2011;46(11):1300-6.

29. Xu C, Yu C, Ma H, Xu L, Miao M, Li Y. Prevalence and risk factors for the development of nonalcoholic fatty liver disease in a nonobese Chinese population: the Zhejiang Zhenhai Study. Am J Gastroenterol. 2013;108(8):1299-304.

30. Sanyal AJ, American Gastroenterological A. AGA technical review on nonalcoholic fatty liver disease. Gastroenterology. 2002;123(5):1705-25.

31. Eslam M, Sarin SK, Wong VW, Fan JG, Kawaguchi T, Ahn SH, Zheng MH, Shiha G, Yilmaz Y, Gani R, et al: The Asian Pacific Association for the Study of the Liver clinical practice guidelines for the diagnosis and management of metabolic associated fatty liver disease. Hepatol Int 2020.

\section{Figures}




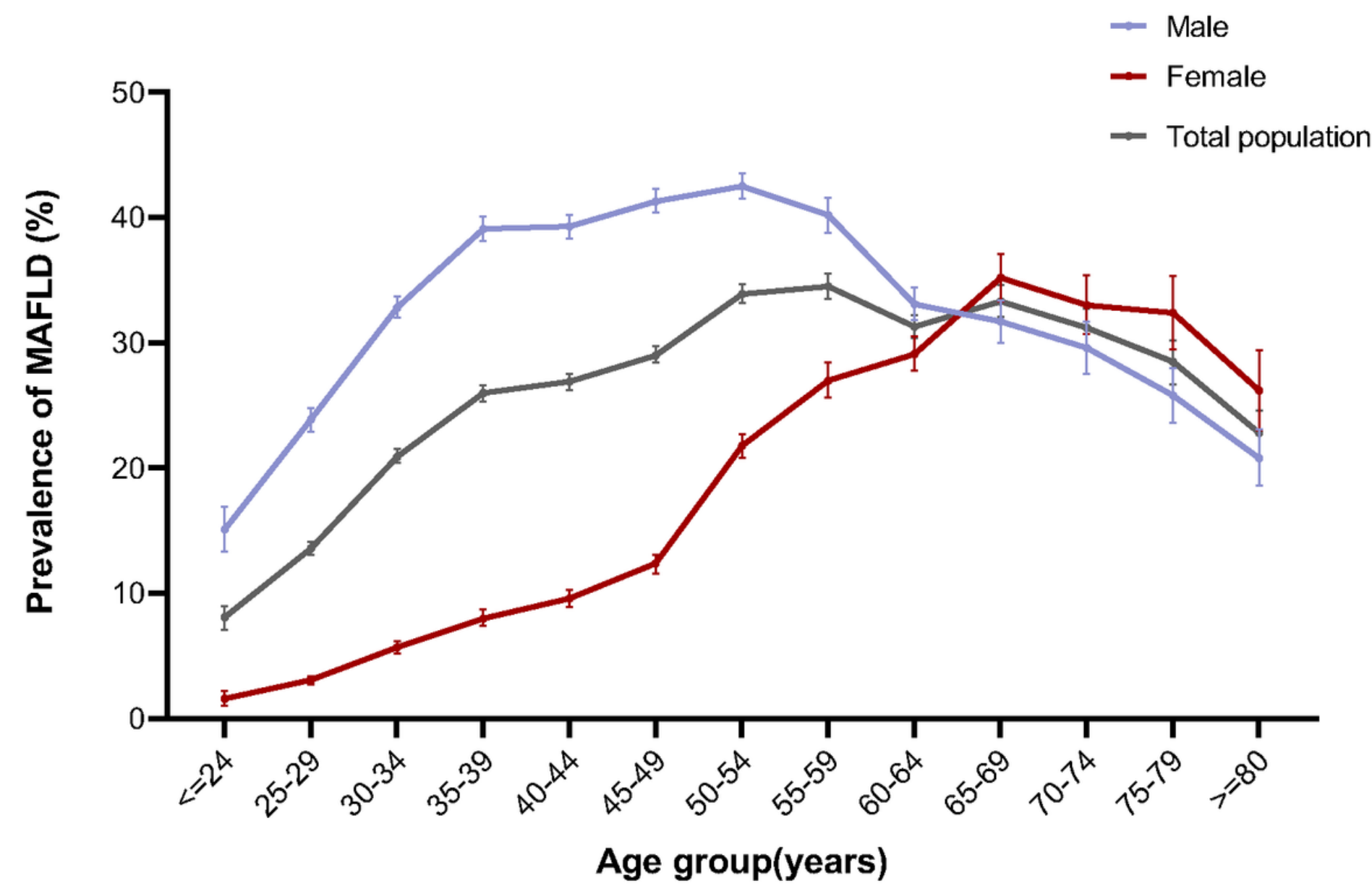

Figure 1

Age-specific prevalence of MAFLD Age-specific prevalence of MAFLD and its 95\% Cls in males, females and total population were calculated. 


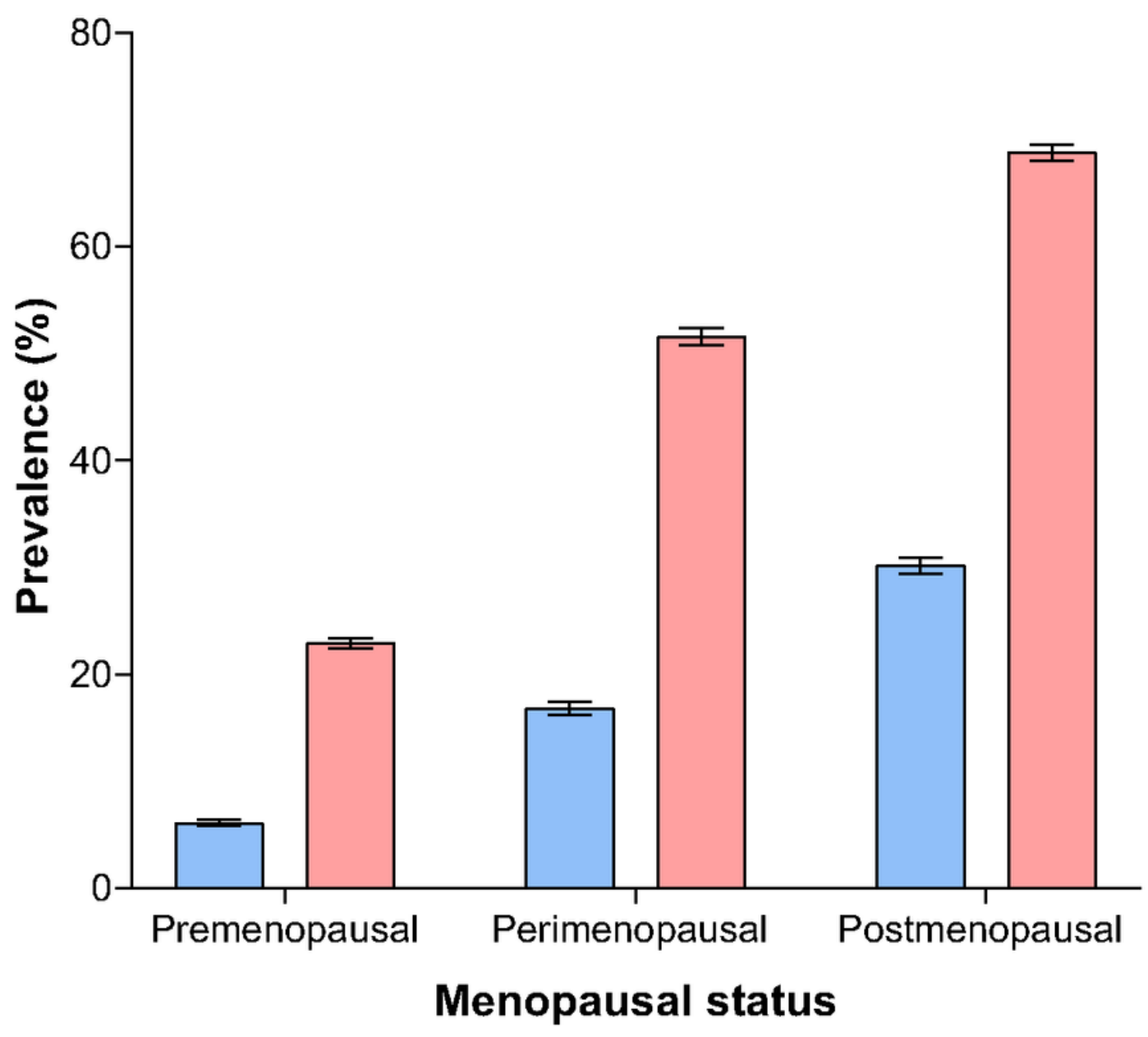

Prevalence of MAFLD Prevalence of dyslipidemia

Figure 2

Prevalence of MAFLD and dyslipidemia in females divided by menopausal status The prevalence of MAFLD and dyslipidemia and its $95 \%$ Cls in females with different menopausal status were calculated. 


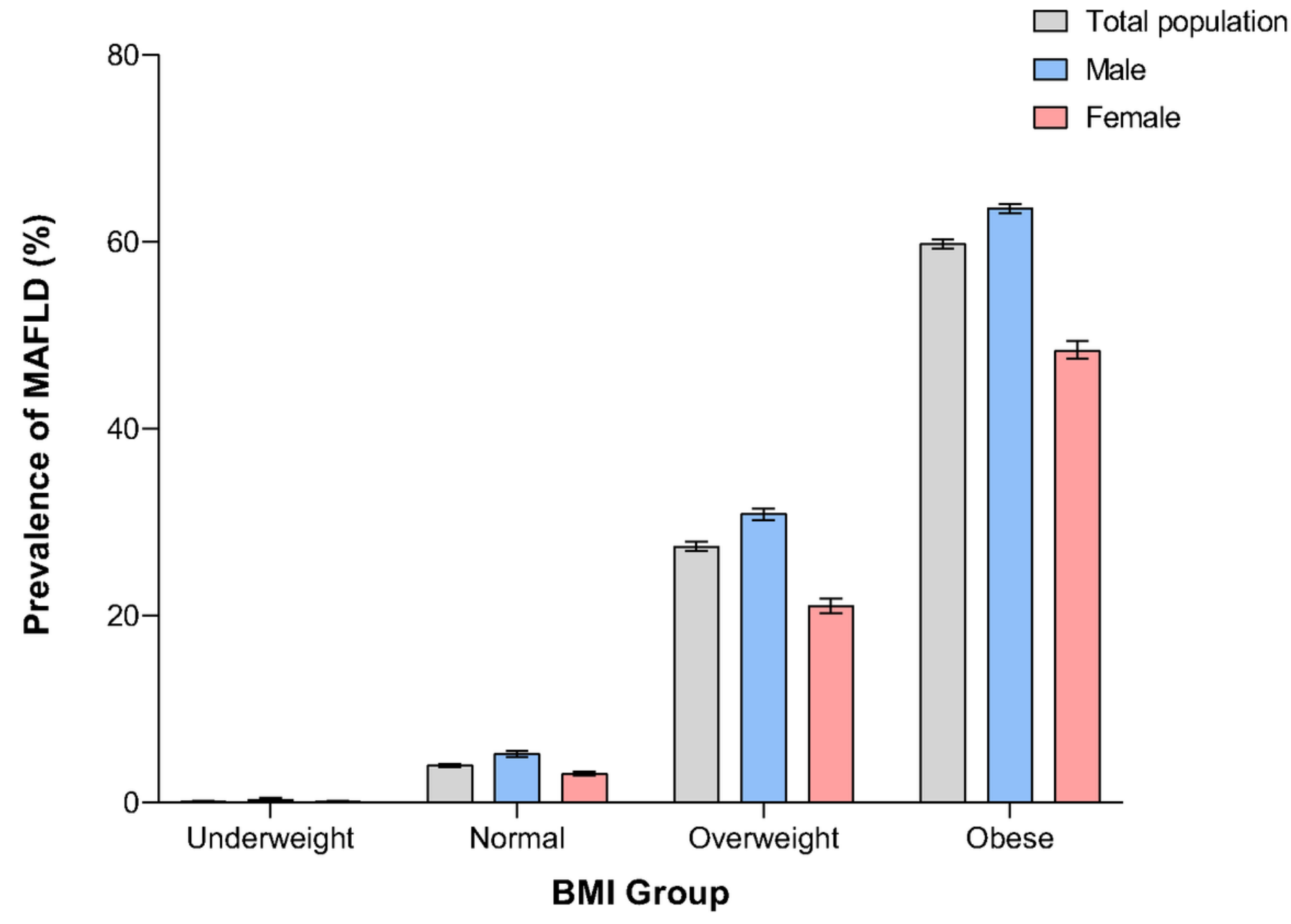

Figure 3

Prevalence of MAFLD in different BMI groups BMI-stratified MAFLD prevalence and its Cls in males, females and total population were calculated. 


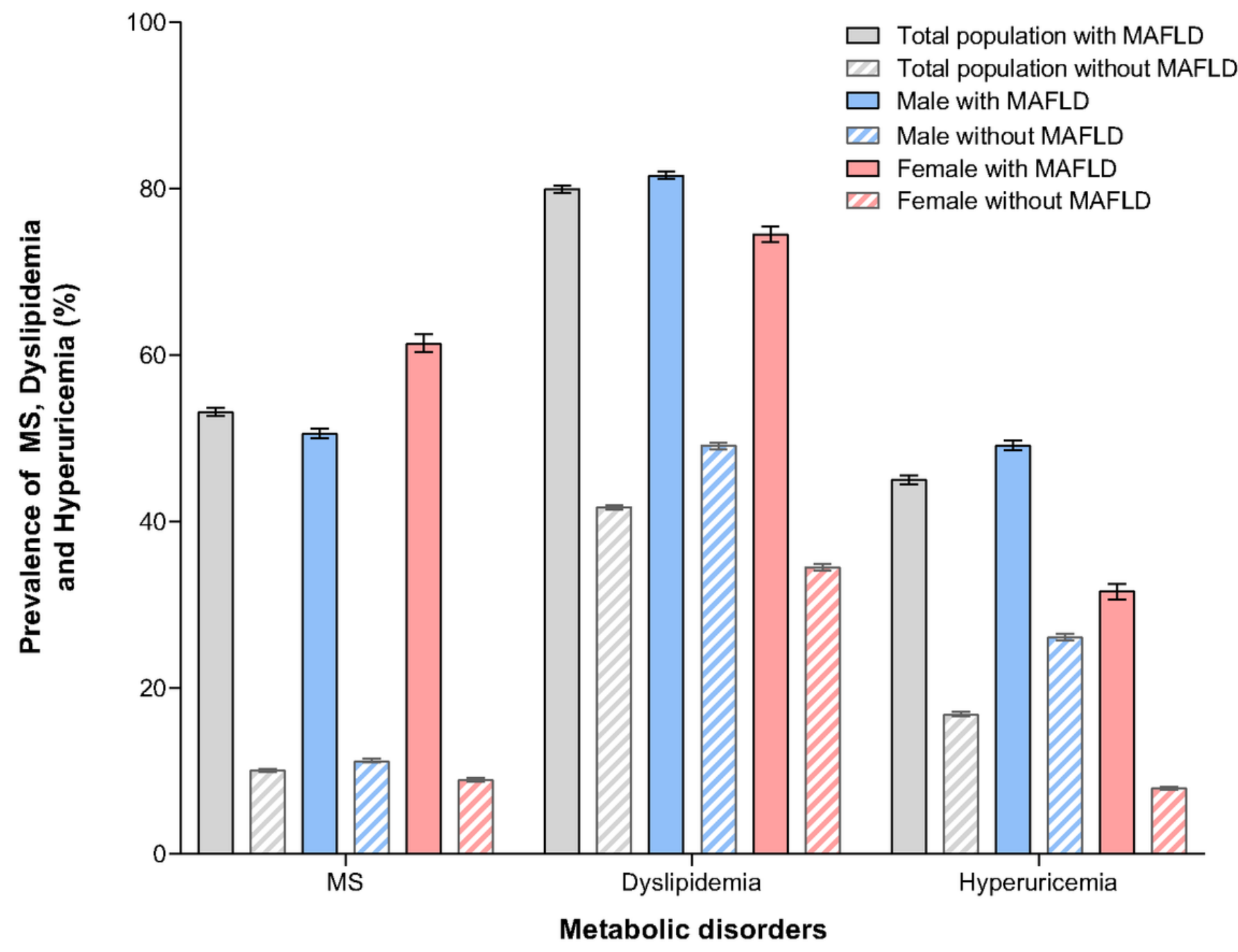

Figure 4

Prevalence of MS, Dyslipidemia and Hyperuricemia in people with and without MAFLD The prevalence of MS, Dyslipidemia and Hyperuricemia and its Cls in people (total population, males and females) with and without MAFLD were calculated. 